\title{
ILKAP Binding to and Dephosphorylating HIF-1 $\alpha$ is Essential for Apoptosis Induced by Severe Hypoxia
}

\author{
Tielong Liu Yonggang Liu $^{\mathrm{a}} \quad$ Jiashi Cao ${ }^{\mathrm{a}} \quad$ Xin $\mathrm{Gao}^{\mathrm{a}} \quad$ Jing Wang ${ }^{\mathrm{a}}$ \\ Guanghui Chen $^{b}$ Yang Wang ${ }^{a}$ Ge Liu ${ }^{a}$ Guangjian Baia Yiwei Hu \\ Jiaxiang Yang ${ }^{\mathrm{a}}$ Lianfeng Dong ${ }^{\mathrm{a}} \quad$ Xinbao Quan ${ }^{\mathrm{a}} \quad$ Weiwei Zou ${ }^{\mathrm{c}}$ \\ ${ }^{a}$ The Spinal Tumor Center, Changzheng Hospital, Second Military Medical University, Shanghai, ${ }^{b}$ Taishan \\ Medical University, Shandong province, 'The Department of Medical Imaging Diagnosis, Changzheng \\ Hospital, Second Military Medical University, Shanghai, China
}

\section{Key Words}

Ilkap • Dephosphorylation • HIF-1 1 - Hypoxia • Apoptosis

\begin{abstract}
Background/Aims: Integrin-linked kinase-associated phosphatase (ILKAP), a serine/threonine phosphatase that belongs to the protein phosphatase $2 \mathrm{C}$ family, has a role in cell survival and apoptosis. Hypoxia-inducible factor $1 \alpha(\mathrm{HIF}-1 \alpha)$ is the key transcription factor in the response to oxygen deficiency in mammals. Direct phosphorylation and dephosphorylation of HIF-1 $\alpha$ affect its function. The present study investigated the role of ILKAP on HIF-1 $\alpha$ dephosphorylation and cell behavior. Methods: HIF-1 $\alpha$ was induced by hypoxia. Physical binding between ILKAP and HIF- $1 \alpha$ was demonstrated by a co-immunoprecipitation assay. HIF- $1 \alpha$ transcriptional activity was investigated using a hypoxia-response element-containing luciferase reporter plasmid. Cell viability was evaluated by a trypan blue dye exclusion assay. ILKAP function was explored by a gain and loss assay with an overexpression plasmid and shRNA infection. Results: ILKAP physically interacted with HIF-1 $\alpha$ and induced its dephosphorylation. Both the HIF-1 $\alpha-p 53$ interaction and apoptosis relied on ILKAP. Conclusion: The results indicated that the ILKAP directly binds and dephosphorylates HIF-1 $\alpha$ and responsible for severe hypoxiainduced cell apoptosis.

\section{Introduction}

Hypoxia-inducible factor 1 (HIF-1), a basic helix-loop-helix transcription factor, is a heterodimer containing HIF- $1 \alpha$ and HIF- $1 \beta$ that plays a crucial role in $\mathrm{O}_{2}$ homeostasis [1]. Under hypoxia, HIF- $1 \alpha$ dimerizes with HIF-1 $\beta$ and binds to a $5^{\prime}-(\mathrm{A} / \mathrm{G}) \mathrm{CGTG}-3^{\prime}$ sequence

T. Liu, Y. Liu and J. Cao contributed equally to this work. 
within hypoxia-responsive elements to activate the transcription of target genes, such as erythropoietin, vascular endothelial growth factor, and various glycolytic enzymes. HIF$1 \alpha$ is involved in many physiological and pathological processes including erythropoiesis, angiogenesis, cell viability, and energy metabolism. HIF- $1 \alpha$ is ubiquitinated and degraded by the proteasome under normoxic conditions [2]. Hypoxia induces HIF-1 $\alpha$ protein by preventing its ubiquitination, and the subsequent phosphorylation of HIF- $1 \alpha$ enhances its transcriptional activity [3]. On the other hand, HIF-1 $\beta$ is expressed constitutively in the nucleus and functions as a common subunit of multiple basic helix-loop-helix family proteins [4]. Thus, HIF-1 transcriptional activity is mainly determined by the HIF-1 $\alpha$ subunit in response to hypoxic and normoxic conditions.

Integrin-linked kinase-associated phosphatase (ILKAP) was identified originally in 1998 as a serine/threonine phosphatase belonging to the protein phosphatase 2C (PP2C) family [5]. ILKAP baits and binds directly to integrin-linked kinase (ILK) [6], which is a serine/threonine kinase that binds directly with the cytoplasmic tail of integrin $\beta 1$ to form an ILK-integrin $\beta 1$ complex [7]. ILKAP binds with the ILK-integrin $\beta 1$ complex to repress the downstream signaling pathway, resulting in blocked cell cycle progression, cell proliferation, and survival, while it promotes cell apoptosis and death [8]. Although the ILKAP catalytic domain shares apparent sequence homology with the PP2C family, its noncatalytic domain differentiates it from other PP2C family members because of a unique sequence that shares no homology with any known proteins. This impressive property of ILKAP indicates that it undergoes specific interactions with other target proteins [9].

In our previous study, we demonstrated that both endogenous and tagged ILKAP are mainly located in the nucleus and its nuclear localization signal is positioned between amino acids 71 and 86, and ILKAP binds with importin $\alpha 1, \alpha 3$, and $\alpha 5$ via Lys78 and Arg79. We also showed that nuclear ILKAP interacts with p90 ribosomal S6 kinase 2 (RSK2), a classic serine/ threonine kinase [10], and inhibits its activity, resulting in the decreased expression of its downstream substrate cyclin D1 [9]. Considering the multiple functions of its phosphatase and the unique sequence of ILKAP, we speculate that RSK2 may not be the sole target protein of ILKAP.

The regulation of HIF- $1 \alpha$ activity includes but not limited to the expression alteration, and the post-translational modification including phosphorylation, ubiquitination and dephosphorylation. Suzuki et al. found that exposure to prolonged and severe hypoxia induces both the phosphorylation and dephosphorylation of HIF-1 $\alpha$. Phosphorylated HIF- $1 \alpha$ is the major form that binds to HIF-1 $\beta$ and is responsible for cell survival, while dephosphorylated HIF- $1 \alpha$ is the major form that binds to p53 and maintains the stabilization of p53 and promotes its subsequent activation, such as apoptosis during severe hypoxia [11]. However, the mechanism for the severe hypoxia-induced dephosphorylation of HIF-1 $\alpha$ is still barely understood and the responsible phosphatase is unknown. In this current study, we found that ILKAP physically interacts with HIF-1 $\alpha$ directly and dephosphorylates HIF- $1 \alpha$. Both the stability and transcriptional activity of HIF- $1 \alpha$ were significantly reduced by ILKAP. Our findings demonstrated that the ILKAP-induced dephosphorylation of HIF- $1 \alpha$ is a crucial mode of regulating HIF- $1 \alpha$ activity and downstream cellular apoptosis, and suggested the important role of ILKAP in hypoxia.

\section{Materials and Methods}

\section{Cell culture and oxygen deprivation}

The tumor cell line A172 was maintained in Dulbecco's modified Eagle's medium with 10\% fetal bovine serum (FBS), $50 \mathrm{U} / \mathrm{mL}$ penicillin, and $50 \mathrm{mg} / \mathrm{mL}$ streptomycin, and cultured in a $37{ }^{\circ} \mathrm{C}$ humidified atmosphere incubator with $5 \% \mathrm{CO}_{2}$. All experiments were performed using the cells under exponential growth and were repeated at least twice.

Oxygen deprivation was performed according to our previous study $[12,13]$. Briefly, the cells were cultured in a modular incubator chamber (Billups-Rothenberg, Del Mar, CA) that had been flushed with a 


\section{Cellular Physiology Cell Physiol Biochem 2018;46:2500-2507

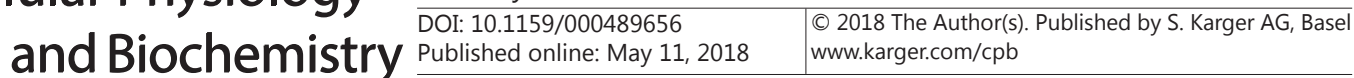

Liu et al.: ILKAP Dephosphorylates HIF-1 $\alpha$ to Promote Hypoxia Induced Apoptosis

mixture of $\mathrm{O}_{2}, \mathrm{~N}_{2}$ (the ratio depended on the experimental design) and $5 \% \mathrm{CO}_{2}$ for $24 \mathrm{~h}$. Hypoxic conditions within the chamber were monitored using a YSI Model 55 Dissolved Oxygen Meter (YSI, Inc., Yellow Springs, $\mathrm{OH})$.

\section{Measurement of cell viability}

A trypan blue dye (Sigma, St. Louis, MO) exclusion assay was performed to determine cell viability. Viable and nonviable cells were counted using a hemocytometer after cellular trypan blue suspension. At least 200 cells were counted for each data point.

\section{Western Blotting}

The cells were lysed on ice for $30 \mathrm{~min}$ in lysis buffer containing $50 \mathrm{mM}$ Tris- $\mathrm{HCl}, \mathrm{pH} 7.4,150 \mathrm{mM}$ $\mathrm{NaCl}, 1 \%$ Nonidet $\mathrm{P}-40$, and $0.1 \%$ sodium dodecyl sulfate (SDS) supplemented with protease inhibitors (10 mg/mL leupeptin, $10 \mathrm{mg} / \mathrm{mL}$ pepstatin A, and $10 \mathrm{mg} / \mathrm{mL}$ aprotinin). For western blot analysis, $15 \mu \mathrm{g}$ sample was resolved by 12\% SDS-polyacrylamide gel electrophoresis (PAGE) and electrotransferred onto a nitrocellulose membrane (Whatman, Piscataway, NJ) [14]. The primary antibodies used were anti-HIF-1 $\alpha$, anti-ILKAP, and anti-p53 from Novus Biologicals (Littleton, CO), and anti-cleaved caspase-3, anti-cleaved caspase-7, and anti-cleaved caspase-8 from Cell Signaling Technology (Danvers, MA) at a dilution of 1:500. To normalize protein loading, an anti- $\beta$-actin (8457; Cell Signaling Technology) antibody was used at a 1:2, 000 dilution. Horseradish peroxidase-conjugated secondary antibodies were used at a 1:2, 000 dilution. Antigen-antibody complexes were visualized using an enhanced chemiluminescence detection system (Millipore, Billerica, MA) as recommended by the manufacturer. The immunoreactive bands were analyzed semi-quantitatively by normalizing band intensity to the background on the scanned films with Quantity One ${ }^{\circledR}$ software (Bio-Rad Laboratories, Inc., Hercules, CA), and relative quantification is indicated below each band in the figures.

\section{Co-immunoprecipitation}

For immunoprecipitation analysis, the cells were lysed in a lysis buffer containing $10 \mathrm{mM}$ HEPES-KOH (pH 7.4), 5 mM MgCl, 142.5 mM KCl, $1 \mu$ M MG-132 (Peptide Institute, Inc., Osaka, Japan), 0.5\% NP-40, 1 mM EGTA, and protease inhibitor cocktail for $10 \mathrm{~min}$ at $4{ }^{\circ} \mathrm{C}$ Equal amounts of proteins were pre-cleared and immunoprecipitated using the anti-ILKAP, anti-HIF-1 $\alpha$, and anti-p53 antibodies, respectively. The immunoprecipitates were then washed with a washing buffer containing $10 \mathrm{mM}$ HEPES-KOH (pH 7.4), 5 $\mathrm{mM} \mathrm{MgCl} 2,142.5 \mathrm{mM} \mathrm{KCl}, 0.5 \% \mathrm{NP}-40$, and $1 \mathrm{mM}$ EGTA, and then resolved by SDS-PAGE and revealed by immunoblot analysis.

\section{Dephosphorylation assay}

As described, HIF-1 $\alpha$ was immunoprecipitated using the anti-HIF-1 $\alpha$ antibody. The immunoprecipitates were washed with phosphatase buffer (PBS) and incubated with 400 U ILKAP (Novus Biologicals), PBS as a negative control, and $400 \mathrm{U} \lambda$ phosphatase (New England BioLabs, Ipswich, MA) as a positive control for $30 \mathrm{~min}$ at $30^{\circ} \mathrm{C}$.

\section{Cell infection}

An ILKAP overexpression plasmid and ILKAP short hairpin RNA (shRNA) expressing adenovirus were purchased from the Han-bio Company (Shanghai, China). Cell infection was performed according to the manufacturer's guidelines. Briefly, the cells were plated for $24 \mathrm{~h}$ prior to infection in 6-well plates. After 24 $\mathrm{h}$ incubation in FBS-free medium for serum starvation, $2 \mu \mathrm{L}$ virus solution with a titer of $1.0 \times 10^{10} \mathrm{PFU} / \mathrm{mL}$ was added to each well, and the cells were incubated at $37{ }^{\circ} \mathrm{C}$ overnight. Hypoxia and respective infection were performed simultaneously at a designed $\mathrm{O}_{2}$ concentration and time schedule.

\section{Statistical analysis}

The results are expressed as the mean \pm standard deviation. Statistical analysis was carried out using one-way analysis of variance (ANOVA) with Tukey's multiple comparisons test or two-way ANOVA with Sidak's multiple comparisons test. P-values less than $0.05,0.01$, or 0.001 were considered statistically significant, very significant, or extremely significant, respectively. 


\section{Cellular Physiology Cell Physiol Biochem 2018;46:2500-2507 and Biochemistry Published onIne: May 11, $2018 \quad \begin{aligned} & \text { DOI: 10.1159/000489656 } 2018 \text { The Author(s). Published by S. Karger AG, Basel } \\ & \text { www.karger.com/cpb }\end{aligned}$}

Liu et al.: ILKAP Dephosphorylates HIF-1 $\alpha$ to Promote Hypoxia Induced Apoptosis

\section{Results}

Hypoxia-induced apoptosis and ILKAP-dephosphorylated HIF-1 $\alpha$

The viability of the tumor cell line A172 (Fig. 1A) was significantly reduced after exposure to hypoxia for a long period of time together with increased levels of apoptosis markers (Fig. 1B). A TUNEL assay showed apoptosis increased as exposure to hypoxia was prolonged (Fig. 1C). HIF-1 $\alpha$ was gradually induced by hypoxia in a time-dependent manner, as shown by western blotting; moreover, the small molecular band of HIF-1 $\alpha$ (lower one) was significantly increased, especially after $48 \mathrm{~h}$, while the slower migrating band (higher one) decreased as exposure to hypoxia was prolonged (Fig. 1D). To explore the mechanism of the two bands of HIF- $1 \alpha$ and the function of ILKAP, we treated HIF- $1 \alpha$ with $400 \mathrm{U}$ ILKAP, PBS as a negative control, and $400 \mathrm{U} \lambda$ phosphatase as a positive control for 30 $\min$ at $30{ }^{\circ} \mathrm{C}$, respectively. We found that the HIF- $1 \alpha$ bands migrated significantly faster in the ILKAP and $\lambda$ phosphatase groups than in the PBS group (Fig. 1E). Thus, the slower migrating band (higher one with a larger molecular weight) was phosphorylated HIF-1 $\alpha$, while the faster band (lower one with a smaller molecular weight) was dephosphorylated HIF- $1 \alpha$. These results confirmed that the phosphorylation status of HIF- $1 \alpha$ was altered during prolonged hypoxia-induced apoptosis, and suggested that ILKAP was responsible for HIF$1 \alpha$ dephosphorylation.

\section{Interactions among ILKAP,} $H I F-1 \alpha$, and p53 during prolonged hypoxia

To explore the interaction between ILKAP and HIF-1 $\alpha$, the cells were treated with hypoxia for $72 \mathrm{~h}$ and lysed immediately. The cell lysates were immunoprecipitated with the anti-ILKAP antibody. The phosphorylated form of HIF-1 $\alpha$ was preferentially immunoprecipitated by the anti-ILKAP antibody (Fig. 2A). A similar procedure was performed to explore the interaction between HIF-1 $\alpha$ and

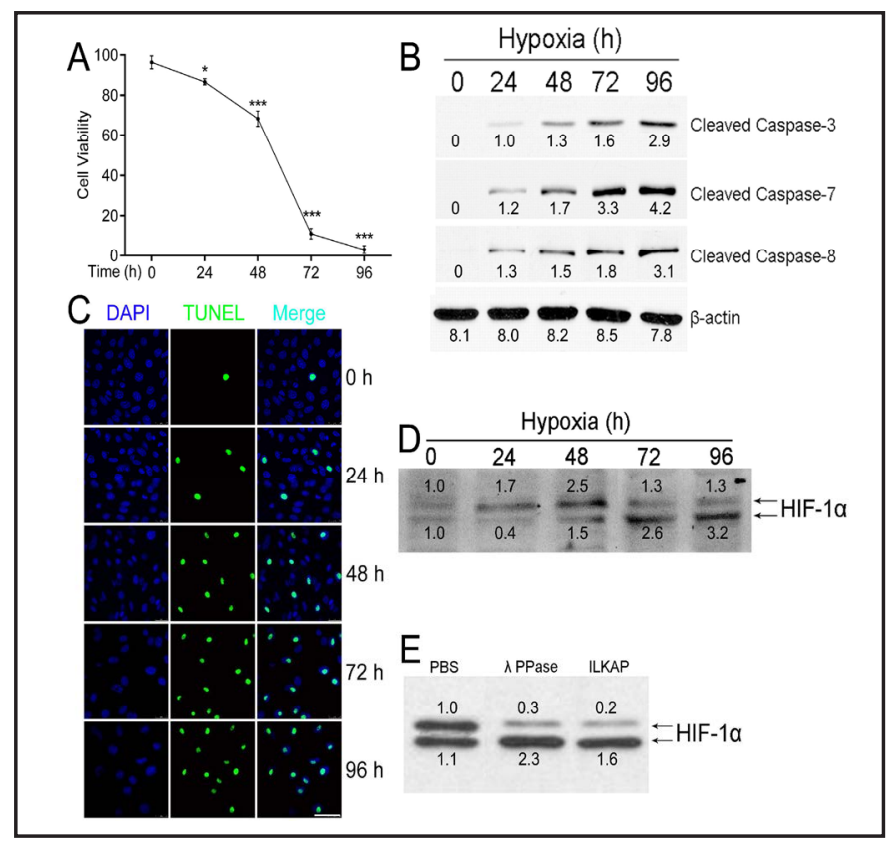

Fig. 1. Hypoxia induced cellapoptosis and ILKAPdephosphorylated HIF-1 $\alpha$. (A) Cell viability was significantly reduced as the hypoxia treatment prolonged. (B, C) The apoptosis markers and TUNEL staining showed increased cell apoptosis. (D) Two bands of HIF$1 \alpha$ were detected and the faster migrated band significantly increased after $48 \mathrm{~h}$. (E) The faster migrated bands were dominant in the ILKAP and $\lambda$ phosphatase treatment groups. The relative quantification was labelled below each band. ${ }^{*}=\mathrm{P}<0.05$; $^{* * *}=$ $\mathrm{P}<0.001$.

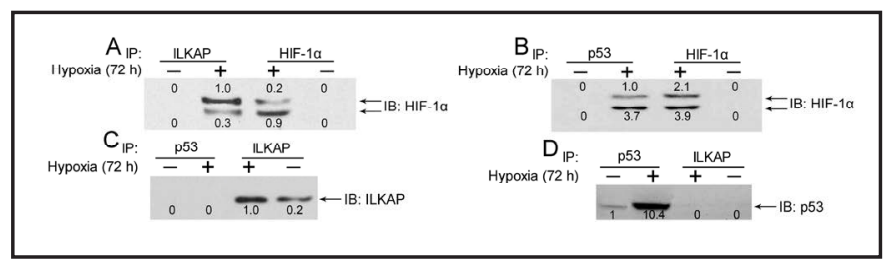

Fig. 2. Interactions among ILKAP, HIF-1 $\alpha$ and p53 in prolonged hypoxia. (A) ILKAP preferentially bound with phosphorylated HIF-1 $\alpha$. (B) p53 preferentially bound with dephosphorylated HIF-1 $\alpha$. (C, D) ILKAP did not directly bind with $\mathrm{p} 53$. The relative quantification was labelled below each band. 


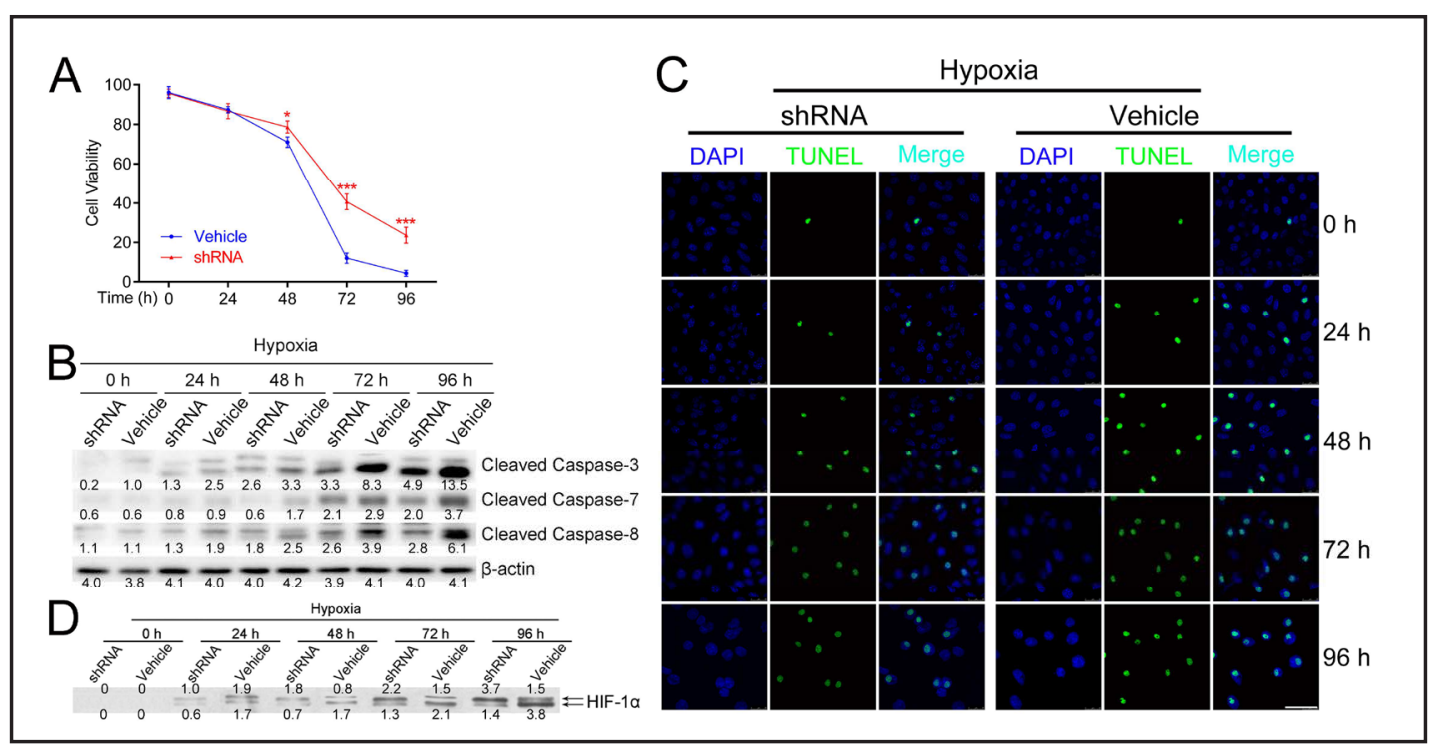

Fig. 3. ILKAP was crucial for prolonged hypoxia induced cell apoptosis. (A) ILKAP shRNA maintained cell viability compared with the vehicle group. (B, C) The apoptosis markers and TUNEL staining showed reduced cell apoptosis in the ILKAP shRNA group compared with the vehicle group. (D) ILKAP shRNA maintained HIF- $1 \alpha$ in the phosphorylated form (slower migrated bands) compared with the vehicle group. The relative quantification was labelled below each band. $*=\mathrm{P}<0.05$; $* * *=\mathrm{P}<0.001$.

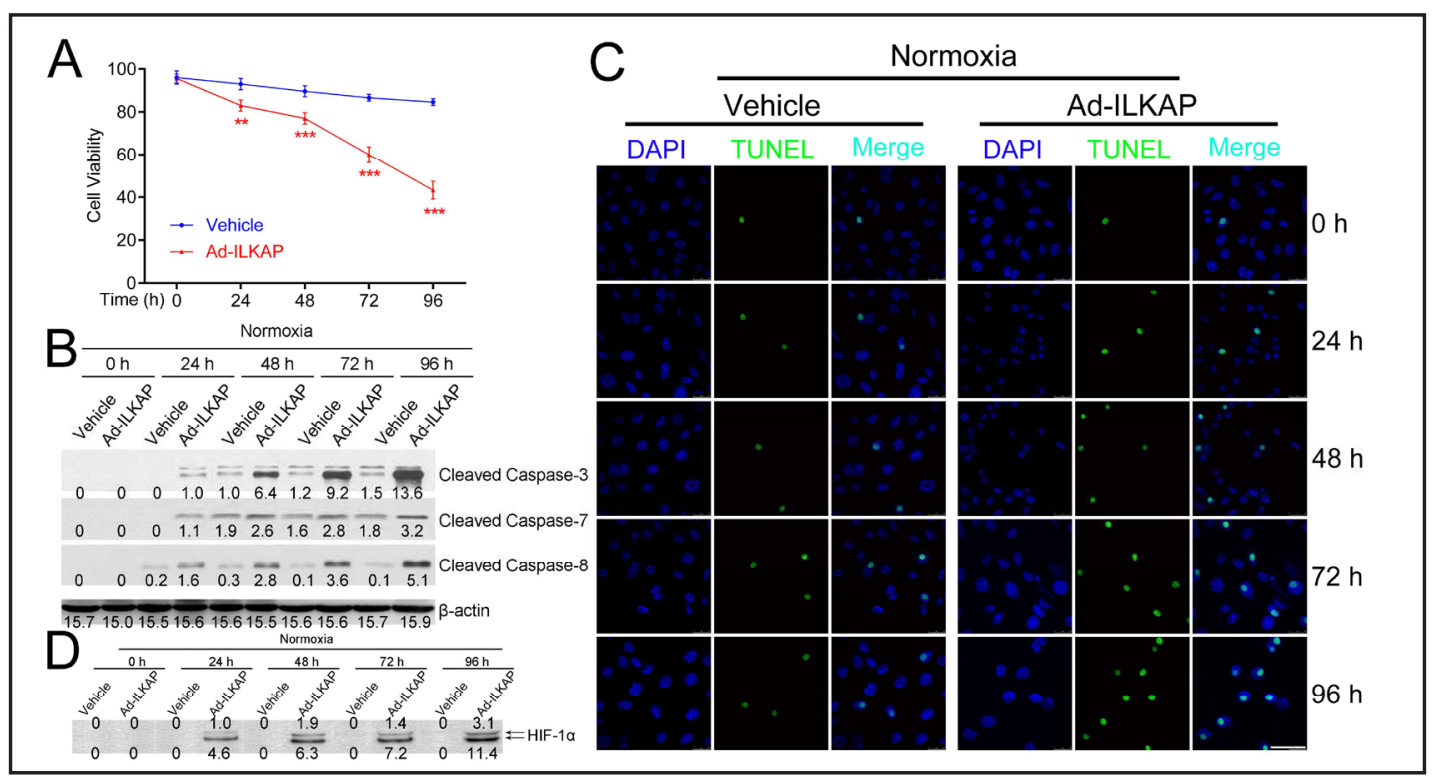

Fig. 4. ILKAP induced cell apoptosis in normoxia situation. (A) Ad-ILKAP reduced the cell viability significantly compared with the vehicle group. (B, C) The apoptosis markers and TUNEL staining showed increased cell apoptosis in the Ad-ILKAP group compared with the vehicle group. (D) Ad-ILKA induced HIF- $\alpha$ expression and dephosphorylation (faster migrated bands) in normoxia situation. The relative quantification was labelled below each band. ${ }^{* *}=\mathrm{P}<0.01 ; * * * \mathrm{P}<0.001$.

p53. However, the dephosphorylated form of HIF-1 $\alpha$ was preferentially immunoprecipitated by the anti-p53 antibody (Fig. 2B). A further assay indicated that ILKAP did not interact with p53 (Fig. 2C and 2D). These results suggested that ILKAP binds directly with phosphorylated HIF- $1 \alpha$ and dephosphorylates it; then, ILKAP and dephosphorylated HIF- $1 \alpha$ are separated, and dephosphorylated HIF- $1 \alpha$ binds directly with p53.

\section{KARGER}


ILKAP is crucial for prolonged hypoxia-induced apoptosis

To explore the role of ILKAP during prolonged hypoxia-induced apoptosis, we performed ILKAP shRNA adenovirus infection during hypoxia treatment. Cell viability was significantly maintained by ILKAP shRNA compared to the vehicle group (Fig. 3A). Apoptosis markers (Fig. 3B) and TUNEL assay (Fig. 3C) showed a significant decrease in apoptosis in the ILKAP shRNA group compared to the vehicle group. The bands showed that HIF-1 $\alpha$ was not significantly transformed to the dephosphorylated form in the ILKAP shRNA group compared with the vehicle group (Fig. 3D). These results suggested that ILKAP is essential for prolonged hypoxia-induced apoptosis.

\section{ILKAP induces apoptosis under normoxia}

To explore further the role of ILKAP in apoptosis, we established an adenovirus ILKAP (Ad-ILKAP) overexpression system and assessed its function under normoxia. Cell viability was significantly reduced in the Ad-ILKAP group in a dose-dependent manner (Fig. 4A). Apoptosis markers (Fig. 4B) and TUNEL assay (Fig. 4C) showed a significant increase in apoptosis in the Ad-ILKAP group compared to the vehicle group. HIF-1 $\alpha$ was not expressed in the vehicle group, but it was detected in the Ad-ILKAP group, and the HIF-1 $\alpha$ bands in the Ad-ILKAP group separated and transformed clearly to the faster migrating band (Fig. 3D). These results indicated that ILKAP induces apoptosis, HIF-1 $\alpha$, and its dephosphorylation under normoxia.

\section{Discussion}

Hypoxia induced p53 and subsequent apoptosis at $0.02 \% \mathrm{O}_{2}$, but not $0.2 \%$, in a mouse embryonic fibroblast study [15] suggesting that the induction of apoptosis is related to the severity of hypoxia. HIF-1 $\alpha$ is responsible for severe hypoxia-induced apoptosis by binding to and stabilizing p53 [16]. Two major forms of HIF-1 $\alpha$ have been identified under severe and prolonged hypoxia: phosphorylated and dephosphorylated HIF-1 $\alpha$. Dephosphorylated, but not phosphorylated, HIF-1 $\alpha$ is closely correlated with p53 induction and the subsequent activation of severe hypoxia-induced apoptosis [11]. However, the phosphatase that regulates the dephosphorylation of phosphorylated HIF-1 $\alpha$ is still unknown.

ILKAP was identified initially in a yeast two-hybrid screen system baited with ILK, and it was considered as a cytoplasmic protein that was involved in the down-regulation of integrin signaling $[6,17]$. The exact substrates of ILKAP are still controversial despite the fact this protein has been studied for many years. Our previous study showed that ILKAP is a nuclear protein, and its nuclear transport is mediated by direct interactions between its nuclear localization signal and importins $\alpha 1, \alpha 3$, and $\alpha 5$. Moreover, we found that apoptosis is induced by ILKAP through the inhibition of RSK2 activity and repression of its downstream substrate cyclin D1 [9]. In the current study, we disclosed another mechanism under hypoxia in which ILKAP is a crucial phosphatase that modulates cell survival and apoptosis through its regulation of HIF-1 $\alpha$. Specifically, ILKAP binds directly to and dephosphorylates HIF-1 $\alpha$ as hypoxia is prolonged. Gain and loss of function assays suggested that ILKAP is crucial for the subsequent induction of apoptosis.

The role of ILKAP in the regulation of cell survival and apoptosis is multispectral. ILKAP can be induced by cellular stresses such as ultraviolet irradiation and ethanol treatment [5]. ILKAP promotes both TNFo-induced AP1 activation and phosphorylation of JNK and p38 in HEK293 cells. However, ILKAP has no effect in HEK293 cells on AP1 activation and JNK or p38 phosphorylation, but further promotes the sustained activation of MAPKs [18]. ILKAP is associated with ASK1 in these cells and activates this kinase by inducing Thr845 phosphorylation. These observations suggest that ILKAP might be a positive regulator of TNF $\alpha$-induced apoptosis. However, it remains to be determined whether ILKAP performs this activation by directly dephosphorylating the inhibitory phosphorylation sites of ASK1, such as Ser83, Ser967, and Ser1034 [18]. Leung et al. found that ILKAP inhibits integrin 
Liu et al.: ILKAP Dephosphorylates HIF-1 $\alpha$ to Promote Hypoxia Induced Apoptosis

signaling by selectively inhibiting GSK3 $\beta$-mediated ILK1 through forming a complex with ILK1 [6]. Oncogenic transformation is also suppressed by ILKAP through the inhibition of ILK1-GSK3 $\beta$ [17]. These studies suggest that ILKAP might regulate the cross-talk between the TNF $\alpha$-induced and integrin-induced signaling pathways by suppressing the former and stimulating the latter to induce the apoptosis of tumor cells [18].

HIF- $1 \alpha$ was identified as a positive factor for tumor growth, and many genes induced by HIF- $1 \alpha$, including angiogenic growth factors and enzymes for glucose metabolism, are upregulated in cancers compared with normal tissues [19]. Thus, HIF-1 $\alpha$ was generally considered as being responsible for carcinogenesis [20]. Moreover, some tumor suppressors, such as p53 [16] and PTEN [21], inhibit HIF-1 $\alpha$ activity. These complicated roles of HIF-1 $\alpha$ might be related to post-translation modifications and its different patterns.

In summary, we revealed that ILKAP directly dephosphorylates HIF- $1 \alpha$ and is responsible for the subsequent p53-related apoptosis. Considering the complicated roles of HIF-1 $\alpha$ and p53, our current study may establish a foundation to investigate the role of different HIF-1 $\alpha$ patterns in many diseases.

\section{Acknowledgements}

This work was supported by the National Natural Science Foundation of China (Grant: 81372874 to TL Liu) and Shanghai Municipal Bureau of Health Research (Grant: 20134335 to WW Zou).

\section{Disclosure Statement}

The authors have no potential conflict of interests.

\section{References}

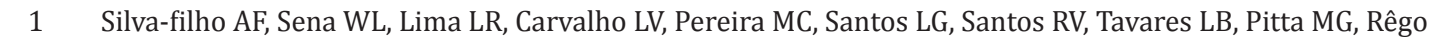
MJ: Glycobiology Modifications in Intratumoral Hypoxia: The Breathless Side of Glycans Interaction. Cell Physiol Biochem 2017;41:1801-1829.

-2 Semenza GL: HIF-1: mediator of physiological and pathophysiological responses to hypoxia. J Appl Physiol 2000;88:1474-1480.

-3 Richard DE, Berra E, Gothié E, Roux D, Pouysségur J: p42/p44 mitogen-activated protein kinases phosphorylate hypoxia-inducible factor $1 \alpha(\mathrm{HIF}-1 \alpha)$ and enhance the transcriptional activity of HIF-1. J Biol Chem 1999;274:32631-32637.

-4 Crews ST: Control of cell lineage-specific development and transcription by bHLH-PAS proteins. Genes Dev 1998;12:607-620.

-5 Tong Y, Quirion R, Shen S-H: Cloning and characterization of a novel mammalian PP2C isozyme. J Biol Chem 1998;273:35282-35290.

-6 Leung-Hagesteijn C, Mahendra A, Naruszewicz I, Hannigan GE: Modulation of integrin signal transduction by ILKAP, a protein phosphatase $2 \mathrm{C}$ associating with the integrin-linked kinase, ILK1. EMBO J 2001;20:2160-2170.

-7 Hannigan GE, Leung-Hagesteijn C, Fitz-Gibbon L, Coppolino MG: Regulation of cell adhesion and anchoragedependent growth by a new beta1-integrin-linked protein kinase. Nature 1996;379:91.

8 Hehlgans S, Haase M, Cordes N: Signalling via integrins: implications for cell survival and anticancer strategies. Biochim Biophys Acta 2007;1775:163-180.

-9 Zhou W, Cao H, Yang X, Cong K, Wang W, Chen T, Yin H, Wu Z, Cai X, Liu T: Characterization of nuclear localization signal in the $\mathrm{N}$ terminus of integrin-linked kinase-associated phosphatase (ILKAP) and its essential role in the down-regulation of RSK2 protein signaling. J Biol Chem 2013;288:6259-6271. 


\section{Cellular Physiology Cell Physiol Biochem 2018;46:2500-2507 \begin{tabular}{l|l} 
DOI: 10.1159/000489656 & $\begin{array}{l}\text { O } 2018 \text { The Author(s). Published by S. Karger AG, Basel } \\
\text { www.karger.com/cpb }\end{array}$
\end{tabular}}

Liu et al.: ILKAP Dephosphorylates HIF-1 $\alpha$ to Promote Hypoxia Induced Apoptosis

10 Doehn U, Gammeltoft S, Shi-Hsiang S, Jensen CJ: p90 ribosomal S6 kinase 2 is associated with and dephosphorylated by protein phosphatase 2C $\delta$. Biochem J 2004;382:425-431.

-11 Suzuki H, Tomida A, Tsuruo T: Dephosphorylated hypoxia-inducible factor 1 [alpha] as a mediator of p53dependent apoptosis during hypoxia. Oncogene 2001;20:5779.

12 Zhang F, Wang J, Chu J, Yang C, Xiao H, Zhao C, Sun Z, Gao X, Chen G, Han Z: MicroRNA-146a induced by hypoxia promotes chondrocyte autophagy through Bcl-2. Cell Physiol Biochem 2015;37:1442-1453.

-13 Chen G, Gao X, Wang J, Yang C, Wang Y, Liu Y, Zou W, Liu T: Hypoxia-induced microRNA-146a represses Bcl2 through Traf6/IRAK1 but not Smad4 to promote chondrocyte autophagy. Biol Chem 2017;398:499-507.

-14 Tong W, Geng Y, Huang Y, Shi Y, Xiang S, Zhang N, Qin L, Shi Q Chen Q, Dai K: In vivo Identification and Induction of Articular Cartilage Stem Cells by Inhibiting NF-кB Signaling in Osteoarthritis. Stem Cells 2015;33:3125-3137.

15 Graeber TG, Osmanian C, Jacks T, Housman DE: Hypoxia-mediated selection of cells with diminished apoptotic potential in solid tumours. nature 1996;379:88.

16 An WG, Kanekal M, Simon MC, Matlepe E: Stabilization of wild-type p53 by hypoxia-inducible factor 1alpha. Nature 1998;392:405.

17 Kumar AS, Naruszewicz I, Wang P, Leung-Hagesteijn C, Hannigan GE: ILKAP regulates ILK signaling and inhibits anchorage-independent growth. Oncogene 2004;23:3454.

18 Tamura S, Toriumi S, Saito J, Awano K, Kudo T, Kobayashi T: PP2C family members play key roles in regulation of cell survival and apoptosis. Cancer Sci 2006;97:563-567.

19 Luo F, Shi J, Shi Q Xu X, Xia Y, He X: Mitogen-Activated Protein Kinases and Hypoxic/Ischemic Nephropathy. Cell Physiol Biochem 2016;39:1051-1067.

20 Weidemann A, Johnson R: Biology of HIF-1 [alpha]. Cell Death Differ 2008;15:621.

-21 Zundel W, Schindler C, Haas-Kogan D, Koong A, Kaper F, Chen E, Gottschalk AR, Ryan HE, Johnson RS, Jefferson AB: Loss of PTEN facilitates HIF-1-mediated gene expression. Genes Dev 2000;14:391-396. 